\title{
Prothèse maxillo-faciale au CHU Lariboisière
}

\section{Maxillofacial prosthesis in Lariboisière Hospital}

\section{MOTS-CLEFS :}

- Prothèse maxillo-faciale, réhabilitation orale, réhabilitation faciale.

\section{KEYWORDS:}

- Maxillofacial prosthesis, oral rehabilitation, facial rehabilitation.

\section{AOS 2013;262:4-9}

DOI: $10.1051 / \mathrm{aos} / 2013202$

(C) EDP Sciences 2013

\section{Résumé}

L'activité de prothèse maxillo-faciale existe ò l'hôpital Lariboisière depuis la Première Guerre mondiale. Ce fut avec le Val-de-Grâce le premier centre de réhabilitation maxillo-faciale. L'Unité fonctionnelle d'Odontologie-Prothèse maxillo-faciale assure la prise en charge des malades du groupe hospitalier, des hôpitaux correspondants ainsi qu'une collaboration avec des praticiens de ville. Son activité recouvre la dermatologie buccale, la chirurgie buccale, l'apnée du sommeil, l'implantologie et plus spécifiquement la prothèse maxillo-faciale.

\author{
Abstract
}

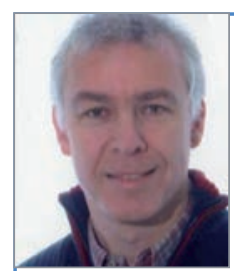

- Didier MAURICE, MCU-PH Paris 7,

Unité Fonctionnelle de PMF, Pôle de Neurosciences - Lariboisière.

Adresse de correspondance: $G$ rue du petit croissant, 91410 Dourdan

Nicolas HELOIRE, AHU Lille, Unité Fonctionnelle de PMF - Lariboisière

Elise KHOURY, DUPMF Paris 7, Unité Fonctionnelle de PMF - Lariboisière

Marc-Christian AUGIER, PH Unité Fonctionnelle de PMF - Lariboisière

Bruno COURRIER, MCU-PH Paris 7, Unité Fonctionnelle de PMF - Lariboisière

\section{INTRODUCTION}

L'activité de prothèse maxillo-faciale à l'hôpital Lariboisière, tant chirurgicale que prothétique, remonte à de nombreuses années puisque c'est au début de la première guerre mondiale que le besoin de la prise en charge des blessures de la face et du crâne s'est imposé aux praticiens. En effet, très rapidement, il apparut que, contrairement aux conflits précédents, les progrès techniques en matière d'armement généraient $95 \%$ de blessures graves; de plus, la stabilisation du front en "guerre des tranchées » où la tête était la partie du corps la plus exposée explique l'importance des blessures de l'extrémité céphalique, tête et cou, qui prendra par extension l'appellation de maxillo-faciale. Ainsi, dans le cadre de la réorganisation du service de santé, dès le 14 novembre 1914, par circulaire ministérielle, sont créés les Centres de réhabilitation maxillo-faciale dont les deux premiers sont situés à Paris (Val-deGrâce sous la direction du Dr Hippolyte Morestin et Lariboisière sous la direction du Dr Pierre Sebileau qui avait succédé en 1901 comme chef du service d'ORL au Dr Gouguenheim, assisté d'Henri Chenet pour la prothèse). Rapidement, deux autres Centres sont ouverts (Lyon sous la direction du Dr Albéric Pont et Bordeaux sous la direction du Dr Cavalie puis Émile Moure) [1].

Le service d'ORL de Lariboisière, appelé alors clinique d'ORL, avait été créé en 1874 par le Dr Émile Isambert qui fut le premier médecin hospitalier à se consacrer à la laryngologie. Il s'agissait alors d'une consultation tolérée, non officielle, installée " dans un local étroit et obscur, d'aspect plutôt misérable ». Deux ans après cette création, la disparition d'Émile Isambert freina le 


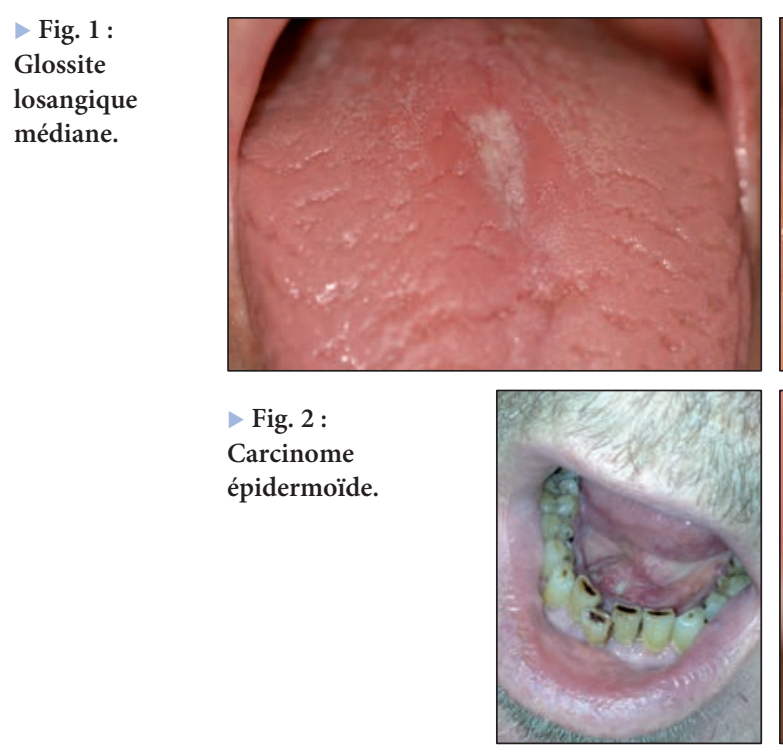

développement de la spécialité ORL. En 1887, Achille Gouguenheim reprit et développa cette activité qui avait périclité. La spécialité était officiellement reconnue par un arrêté en novembre 1897 [2]. Parallèlement à l'activité spécifique ORL, la prothèse maxillo-faciale n'a cessé d'être pratiquée depuis Sebileau et Chenet. Ainsi se sont Ponroy, Psaume, Rigault, Voreaux et Vignon qui ont successivement assuré la direction de cette activité bien que, comme à l'origine du service ORL, l'existence de cette unité soit restée non officielle jusqu'au début janvier 2012, date à laquelle l'Unité fonctionnelle d'Odontologie/Prothèse maxillo-faciale intégrée dans le pôle neurosensoriel/tête et cou a été officiellement créée et reconnue.

Cette Unité a pour vocation d'une part la prise en charge de l'ensemble des malades hospitalisés sur les sites des hôpitaux Lariboisière et Fernand Vidal adressés par les différents services pour des soins de chirurgie buccale et de dermatologie buccale (médecine interne, cardiologie, rhumatologie, orthopédie, gériatrie, urgence et bien entendu l'ORL), d'autre part de recevoir les malades adressés par les hôpitaux extérieurs dans le cadre d'une collaboration chirurgico-prothétique maxillo-faciale et implantaire.

\section{DIFFÉRENTS TYPES D'ACTIVITÉ DE L'UNITÉ}

L'activité d'odontologie chirurgicale consiste dans le dépistage des foyers infectieux et la mise en état de la sphère orale dans le cadre des diverses pathologies des grands systèmes : cardiologie, orthopédie, médecine interne, ORL. À côté de cette activité, la spécificité de l'Unité repose sur la prise en charge des malades nécessitant une réhabilitation prothétique maxillo-faciale en interface avec l'ORL de Lariboisière mais également avec divers services parisiens extérieurs tant Chirurgie cervico-faciale (CCF) que Chirurgie maxillo-faciale (CMF). Cette Unité est avec le service de Stomatologie et Chirurgie maxillo-faciale de la Pitié-Salpêtrière

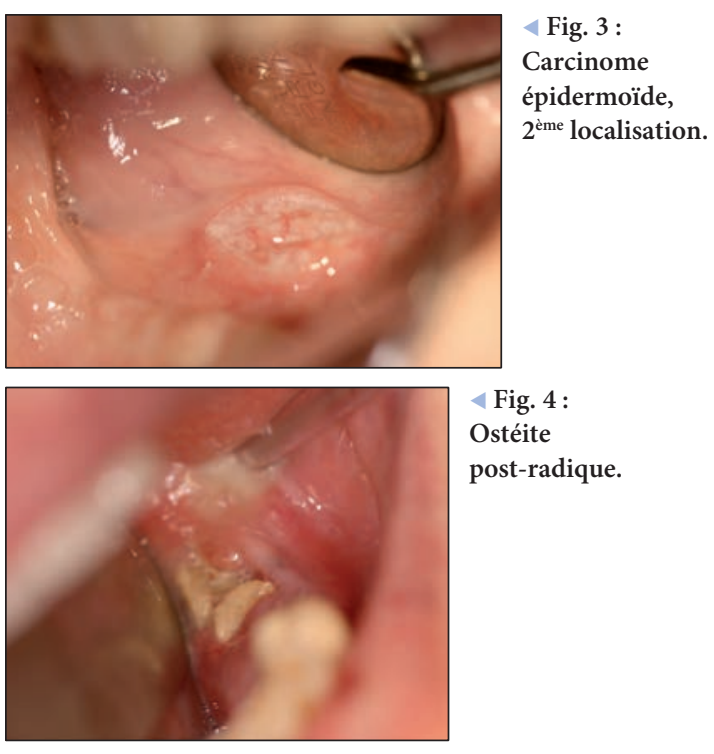

l'un des deux centres de l'AP-HP ayant cette activité spécifique. La prise en charge concerne aussi bien les réhabilitations endo-orales qu' extra-orales suite à de la traumatologie, de la cancérologie ou en phase préparatoire d'une chirurgie orthognatique chez des sujets présentant un édentement partiel ou total. L'unité assure également la réalisation d'orthèses d'avancée mandibulaire dans le cadre des SAOS avec l'Unité du Sommeil et la phase chirurgicale de l'implantologie dentaire (préparation du site par comblement et pose) en accord et à la demande de praticiens de ville.

\section{Dépistage}

Une consultation hebdomadaire de dermatologie buccale est assurée permettant aux praticiens de ville de référer leurs patients en cas de doute ou de difficulté diagnostique sur les pathologies de la muqueuse buccale. Cette consultation participe ainsi à diagnostiquer des pathologies bénignes (fig 1), des pathologies malignes (fig 2). De même pour les malades suivis pour une réhabilitation prothétique maxillo-faciale, l'examen des muqueuses permet de déceler les récidives ou $2^{\mathrm{e}}$ localisations (fig 3 ), ou complications des traitements curatifs tels que l'ostéite post-radique suite à une radiothérapie externe (fig 4) [3].

\section{Traumatologie}

Si l'apparition des plaques de synthèse a fait évoluer le traitement des fractures maxillo-faciales vers la prise en charge chirurgicale aux dépens de la prise en charge orthopédique, il reste des localisations relevant du champ d'activité de la PMF (ex. : traitement orthopédique des fractures de l'ATM). Il n'est d'ailleurs pas inepte d'envisager une prise en charge orthopédique de ces fractures. Par exemple une fracture para-médiane avec déplacement peut parfaitement être traitée par procédé orthopédique ne nécessitant aucune anesthésie générale ni chirurgie, évitant ainsi le risque tant anesthésique que d'atteinte radiculaire ou nerveuse lors de la pose des vis. Lors d'un déplacement, la pose 
Fig. 5 :

Trait de fracture entre $32 / 33$.

Fig. 7 : Réduction puis contention élastique.

Pig. 9 : Résultat à la dépose des arcs de contention.
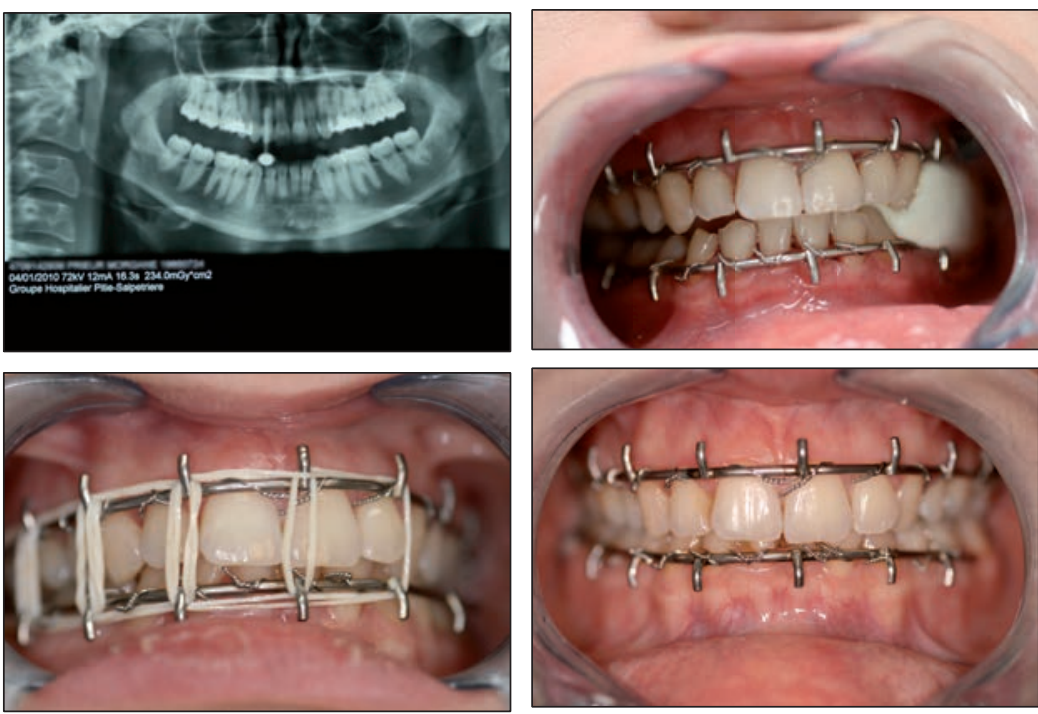

4 Fig. 8 :

Occlusion en fin de contention.

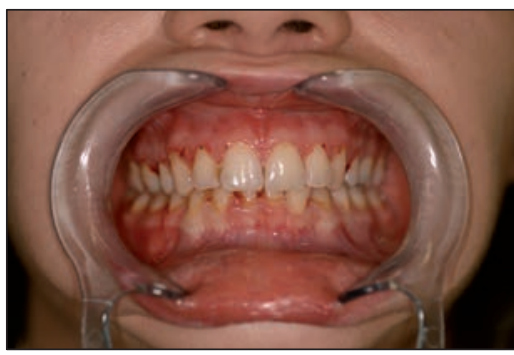

de deux arcs permet d'obtenir une réduction par traction élastique avec cale puis contention après avoir obtenu l'occlusion satisfaisante (fig. 5, 6, 7, 8, 9) [4].

\section{Apnée du sommeil}

L'Unité d'Odontologie-Prothèse maxillo-faciale participe à la prise en charge par orthèse d'avancée mandibulaire des apnées obstructives du sommeil lorsque l'indication est posée par le médecin spécialiste (pneumologue ou ORL). Cette prise en charge consiste en la prise d'empreinte, le titrage de l'orthèse jusqu'à validation de l'efficacité de l'orthèse après polygraphie de contrôle (fig. 10, 11, 12).

\section{Réhabilitation endo-orale}

Elle fait suite à des étiologies traumatiques (accident de la voie publique, balistique), carcinologiques, ortho- gnathiques ou dans le cadre de résorption majeure des bases osseuses maxillaire et/ou mandibulaire. Les réhabilitations prothétiques peuvent nécessiter une phase chirurgicale préparatoire par chirurgie soustractive (extractions, exérèse de kyste) ou additive (greffe osseuse, implants).

\section{Implantologie}

La partie chirurgicale de l'implantologie n'est abordée qu'après élaboration du plan de traitement avec le confrère de ville lors des réhabilitations par prothèse fixée, l'Unité n'ayant pas vocation à la réalisation des soins et prothèses fixées. En cas de prothèse amovo-inamovible ou de prothèse amovible à complément de rétention implantaire, celle-ci peut être faite au sein de l'Unité. Il peut alors être nécessaire préalablement à la pose implantaire de prévoir une prise de greffon soit dans la cavité buccale, soit en dehors de la cavité buccale, nécessitant la collaboration d'un chirurgien. (fig. 13, 14, 15, 16) [5].

\section{Division vélo-palatine}

Les séquelles des divisions vélo-palatines chez l'adulte nécessitent suivant la situation de départ une collaboration chirurgico-prothétique chez des patients présentant un édentement partiel ou total associé à

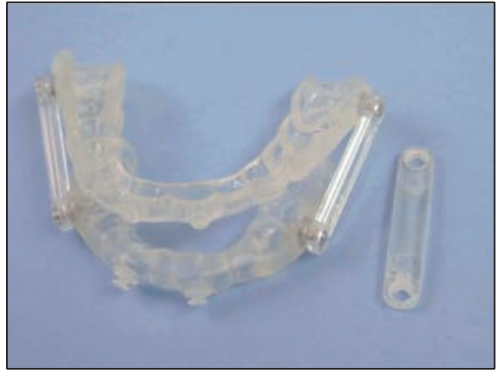

$\triangle$ Fig. 10 :

Orthèse d'avancée mandibulaire.

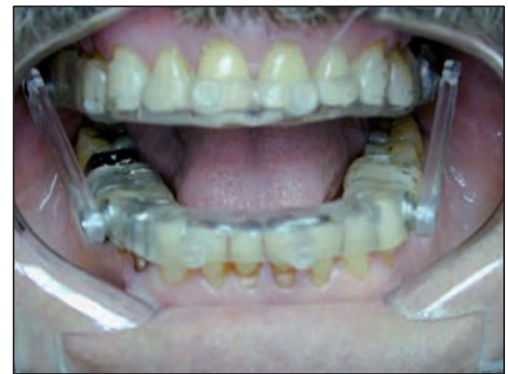

$\triangle$ Fig. 11 :

Situation de l'orthèse en bouche.

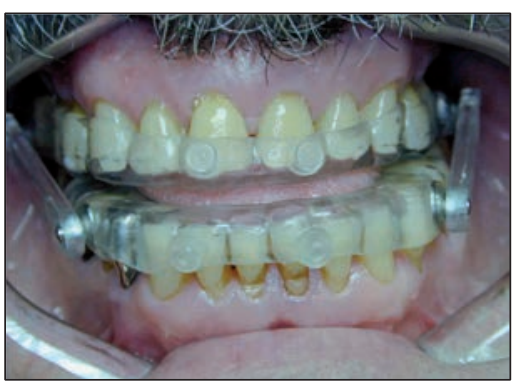

$\triangle$ Fig. 12 :

Le maximum de propulsion est obtenu en bouche fermée. Les plots antérieurs permettent de tendre des élastiques pour favoriser cette fermeture. 
Crête mandibulaire étroite; élargissement avec prélèvement osseux autogène postérieur et pose 2 implants dans le même temps

Fig. 13:

Volet sinusien.
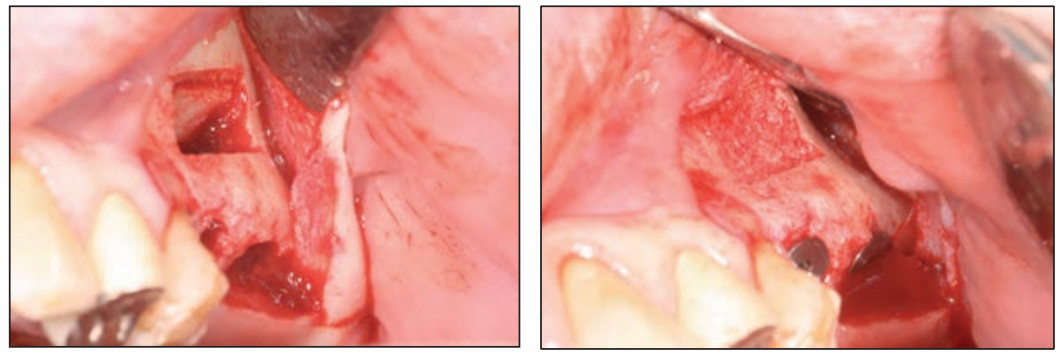

$\varangle$ Fig. 14 :

Pose implantaire

et comblement

dans le même

temps opératoire.

Fig. 15 :

Éclatement de la Crête mandibulaire étroite :

élargissement avec prélèvement osseux autogène postérieur et pose de deux implants dans le même temps opératoire. Eclatement de la crête mandibulaire par volet vestibulaire.
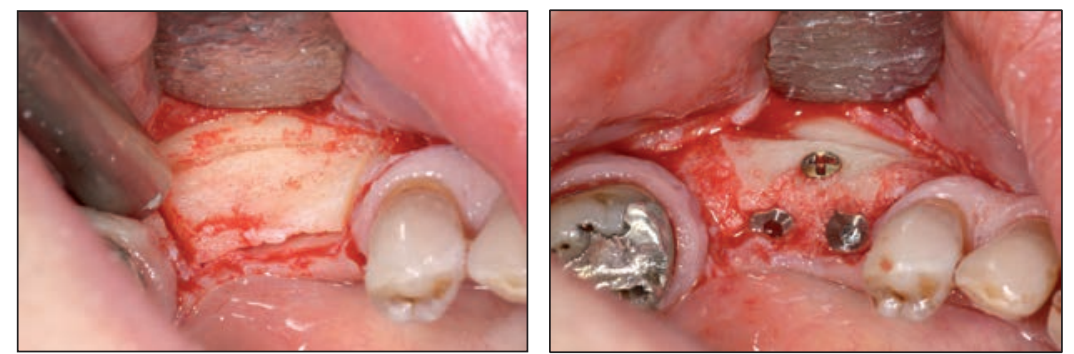

$\varangle$ Fig. 16 :

Crête mandibulaire étroite : élargissement avec prélèvement osseux autogène postérieur et pose de deux implants dans le même temps opératoire. Comblement et synthèse du volet vestibulaire.

une rétrognathie et/ou endognathie. Si les corrections chirurgicales ont été effectuées préalablement, la prothèse permettra de compenser l'édentement et les communications bucco-nasales (fig 17,18 ) et/ou vélaires ; la réhabilitation prothétique peut être initiale ou être un renouvellement de prothèse existante (fig 19, 20, 21) [6].

\section{Pertes de substance maxillaires}

En cas de pertes de substance d'origine traumatique, la prothèse maxillo-faciale sera nécessaire en complément de la chirurgie pour assurer une contention ou le plus souvent dans un second temps pour restaurer une fonction et une esthétique que la chirurgie n'a pu redonner. La coopération chirurgico-prothétique est là encore primordiale pour un résultat optimal, la prothèse maxillo-faciale pouvant intervenir comme guide cicatriciel avant de restaurer les fonctions et l'esthétique.

Lors des pertes de substance d'origine carcinologique, la prothèse peut être immédiate, transitoire et d'usage. La prothèse immédiate permet un résultat optimal pour le malade en étant vu avant l'intervention. Elle est adaptée durant la période de cicatrisation permettant de préparer le lit de la prothèse d'usage. La prothèse maxillo-faciale sera conçue suivant le cas clinique avec
Fig. 17 :

Séquelle de DVP chez un adulte jeune.
Fig. 18 : Obturateur souple en bouche.
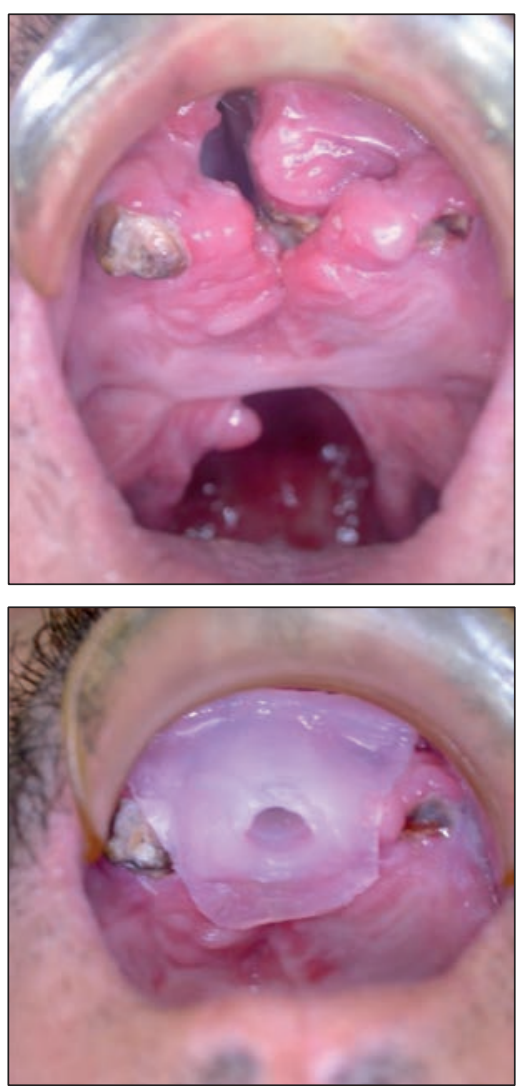

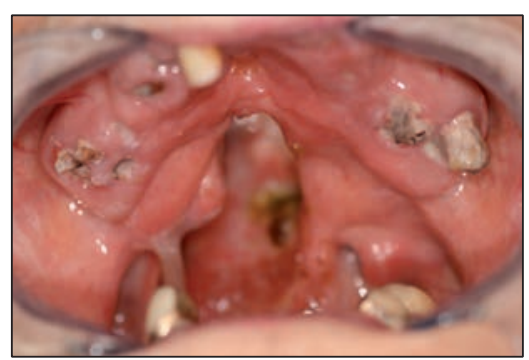

$\triangle$ Fig. 19:

Séquelle de DVP

chez une patiente

de 99 ans.

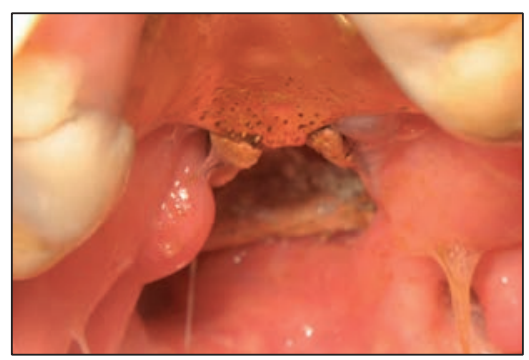

4 Fig. 20 :

Prothèse vélaire

ancienne en

situation.

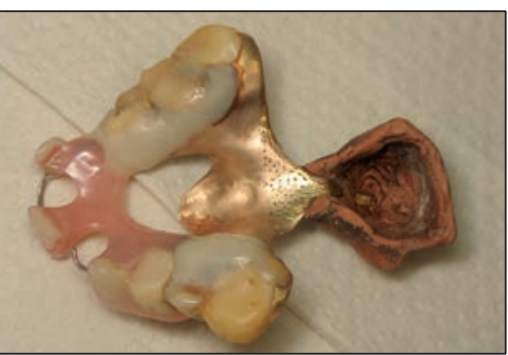

$<$ Fig. 21 :

Prothèse vélaire:

châssis or et

obturateur en

vulcanite. 


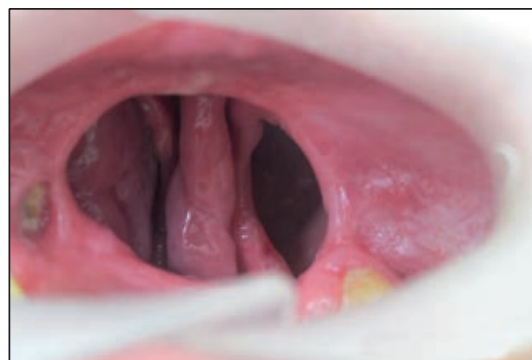

$\triangle$ Fig. 22 :

Perte de substance maxillaire en fin de temporisation.

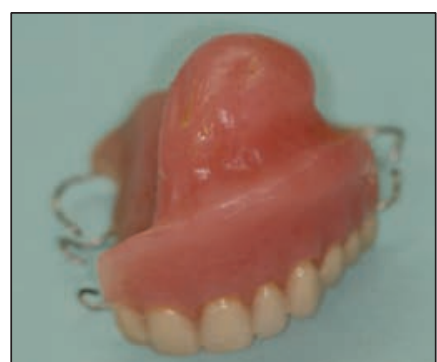

$\triangle$ Fig. 23 :

Prothèse transitoire avec obturateur associé.

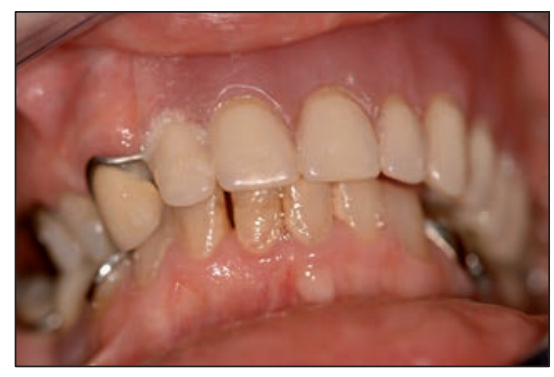

$\triangle$ Fig. 24 :

Perte de substance maxillaire antérolatérale stabilisée.

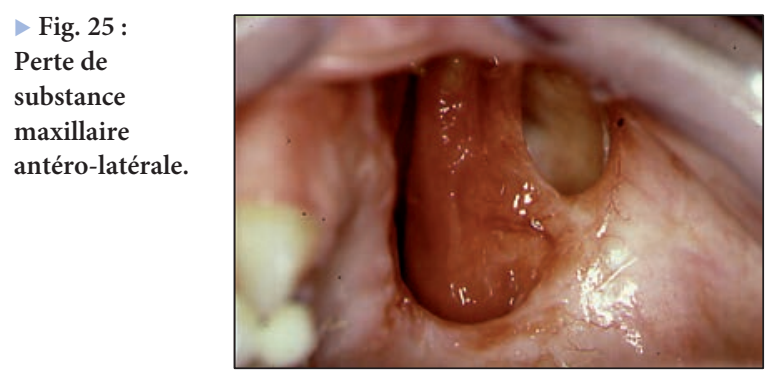

Fig. 27 :

Prothèse

dentaire avec

obturateur

souple

dissocié.
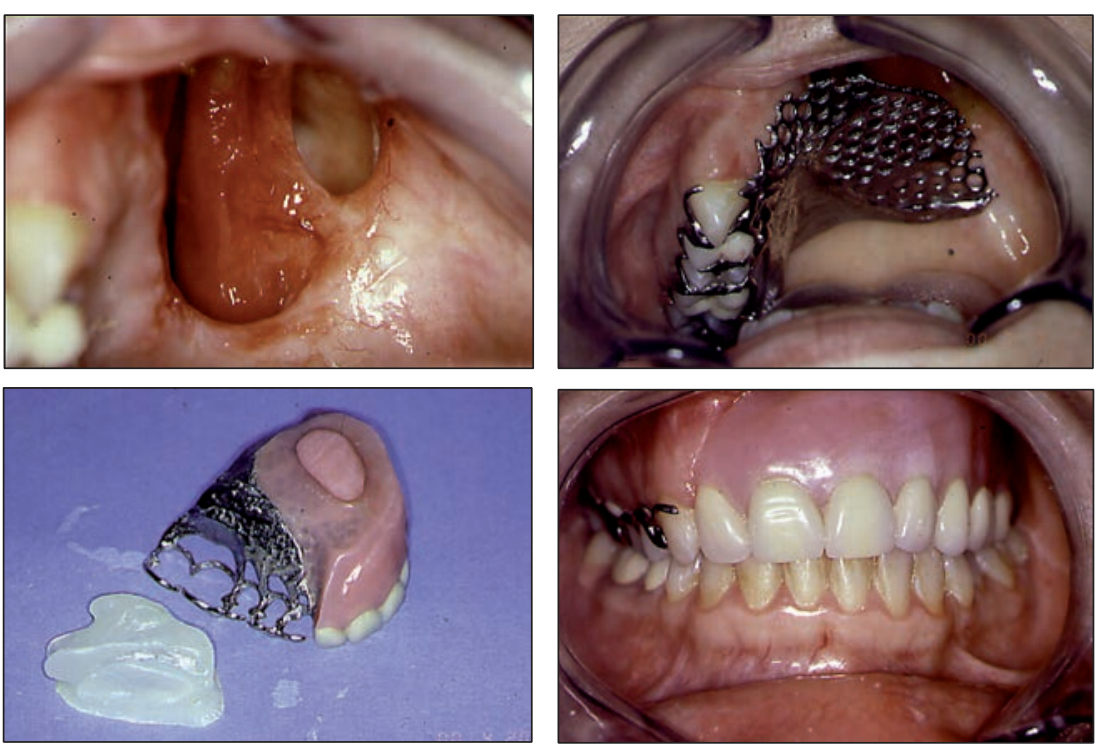

$>$ Fig. 26 :

Châssis métal

de la prothèse.

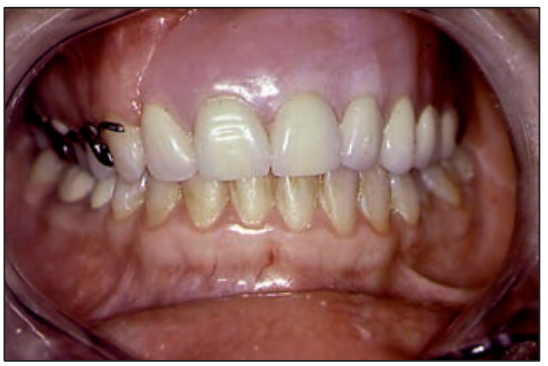

$\triangle$ Fig. 28 :

Prothèse en

bouche.

un obturateur solidaire de la prothèse (fig $22,23,24$ ) ou dissocié de celle-ci (fig 25, 26, 27, 28) ; ceci est rendu plus aisé à gérer par la présence de l'unité au sein du pôle neurosensoriel tête et cou ; de plus, ceci permet la participation aux RCP (Réunions de Concertation Pultidisciplinaires) durant lesquelles les dossiers des malades sont discutés, nous permettant de nous inscrire dans la chronologie de prise en charge thérapeutique.

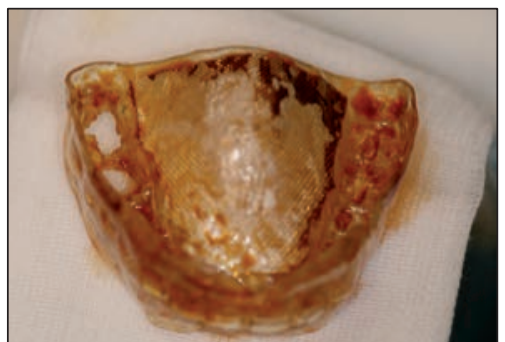

$\triangle$ Fig. 29:

Guide chirurgical basé sur le projet prothétique.

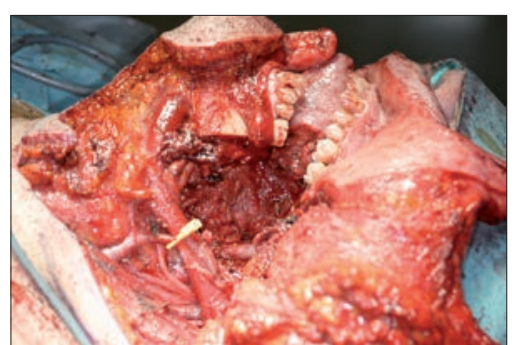

$\triangle$ Fig. 32 :

Exérèse mandibulaire.

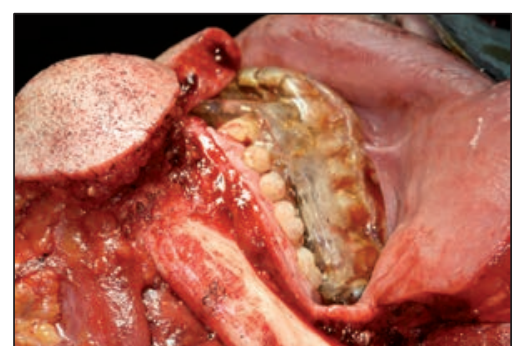

$\triangle$ Fig. 30 :

Guide positionné sur l'arcade maxillaire.

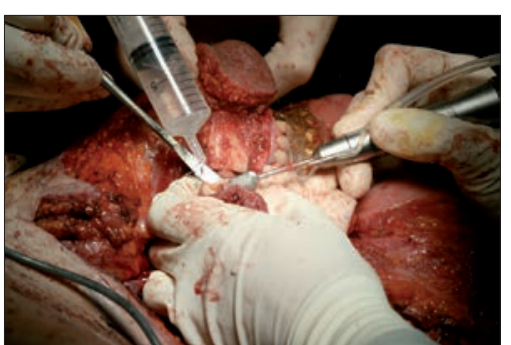

Fig. 33 :

Préparation du greffon suivant

le couloir prothétique.

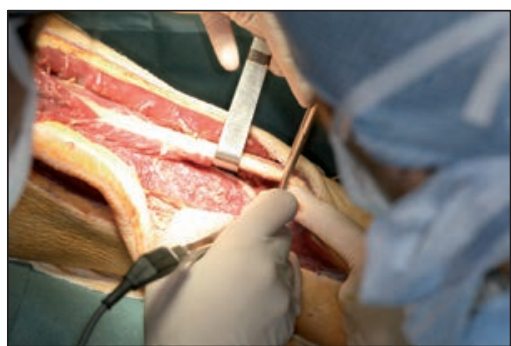

Fig. 31 :

Prélèvement du greffon de fibula.

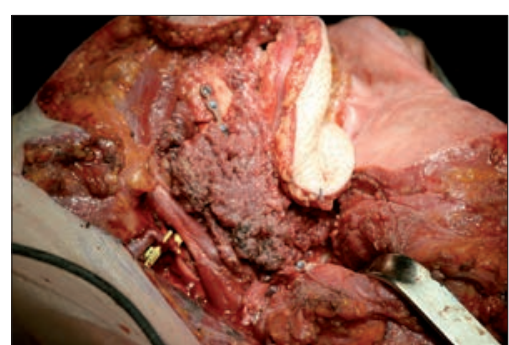

$\triangle$ Fig. 34 :

Ostéosynthèse du greffon de fibula sur la mandibule native suivant le couloir prothétique. 


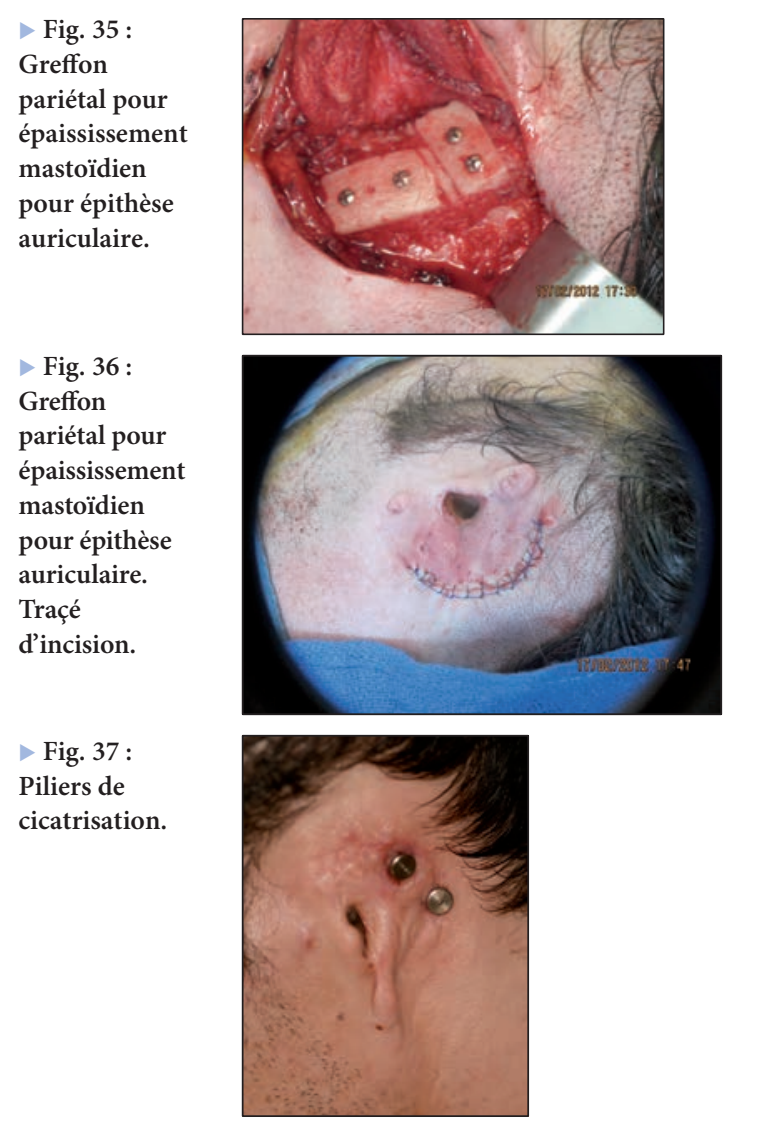

\section{Pertes de substance mandibulaires}

Les pertes de substance mandibulaires sont toujours des réhabilitations difficiles à gérer. Les pertes non interruptrices du type pelvimandibulectomie intéressant l'os alvéolaire respectent l'arc mandibulaire et sont donc assez aisées à réhabiliter. En cas de chirurgie interruptrice non reconstruite, une rééducation précoce est à prévoir et une prothèse guide doit être envisagée si la mandibule part en latéralité. En cas de reconstruction par greffe osseuse, le modelage de celle-ci est fait suivant le projet prothétique de façon à s'inscrire dans le couloir prothétique de la future réhabilitation. Le guide chirurgical est positionné en prenant en référence l'occlusion, le greffon est ensuite positionné suivant l'intrados prothétique du guide (fig 29, 30, 31, 32, 33, 34).

\section{Réhabilitation extra-orale}

Il s'agit des reconstructions prothétiques auriculaires, orbito-palpébrales ou nasales faisant suite à des étiologies traumatique ou carcinologique pour lesquelles l'option de la reconstruction chirurgicale n'a pas été retenue. Elles peuvent aussi intervenir à titre transitoire en attendant une reconstruction chirurgicale [7]. La réhabilitation prothétique désormais est souvent précédée d'une préparation chirurgicale à visée rétentive par implants; un apport osseux peut alors être nécessaire. La mise en place des greffons osseux et des implants est le plus souvent réalisée sous anesthésie générale après validation du projet prothétique à partir duquel un guide radio-chirurgical est conçu

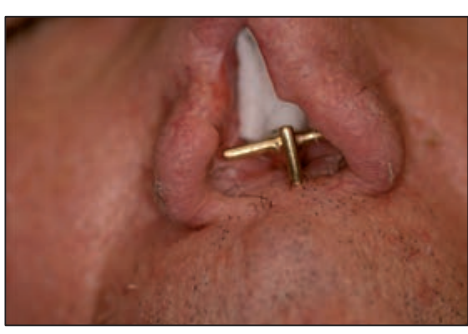

Fig. 38 :
Barre de
rétention sur
implant pour
épithèse nasale.

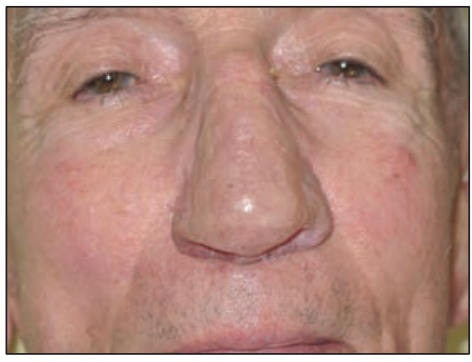

4 Fig. 39:

Epithèse nasale

clippée sur la

barre.

(fig 35, 36, 37). Cette étape préparatoire nécessite une collaboration chirurgico-prothétique entre chirurgiens maxillo- ou cervico-faciaux et odontologistes maxillo-faciaux.

La phase prothétique quant à elle peut ne nécessiter qu'une anesthésie locale lors de la découverte implantaire ; les étapes d'empreinte, d'essayage et de pose se faisant au fauteuil comme en prothèse implanto-portée endo-orale (fig 38, 39).

\section{CONCLUSION}

À côté d'une activité d'odontologie hospitalière permettant la prise en charge des malades du groupe hospitalier et ceux des hôpitaux correspondants ainsi que des patients de correspondants de ville en chirurgie et implantologie, l'Unité fonctionnelle d'Odontologie-PMF a une activité particulière de prothèse maxillo-faciale endo- et extra-orale permettant aux patients de retrouver une fonction, une esthétique et de pouvoir se réinsérer socialement.

\section{Bibliographie}

[1] Monestier M. Les gueules cassées : les médecins de l'impossible. Paris : Le Cherche Midi, 2009.

[2] Legent F. La naissance de l'ORL en France, Paris : Medica, Histoire de la Santé, BIU Santé, 2005.

[3] Kuffer R, Lombardi T, Husson-Bui C, Courrier B, Samson J. La muqueuse buccale : de la clinique au traitement. Paris : Med'com, 2009.

[4] Rigault A, Voreaux P. Traitement orthopédique des traumatismes maxillo-faciaux. Paris : Masson et Cie, Julien Prélat, coll. Association d'enseignement odonto-stomatologique, 1971.

[5] Beumer J, Lewis SG. The Branemark implant system: clinical and laboratory procedures. St Louis: Ishiyaku Euroamerica Inc., 1989.

[6] Benoist M. Réhabilitation et prothèse maxillo-faciales. Paris : Julien Prélat, 1978.

[7] McKinstry RE. Fundamentals of facial prosthetics, Arlington : ABI Professional Publications, 1995. 